\title{
Kann Komplementärmedizin einen aktiven Beitrag zur Prävention von Ůbergewicht leisten?
}

\author{
Ana Paula Simões-Wüst ${ }^{\mathrm{a}, \mathrm{b}}$ \\ a Forschungsabteilung, Paracelsus-Spital Richterswil, Richterswil, Schweiz \\ ${ }^{b}$ Bereich Klinische Forschung, Klinik Arlesheim AG, Arlesheim, Schweiz
}

\section{Schlüsselwörter \\ Übergewicht · Prävention · Nutzung von Komplementärmedizin . Biologische Ernährung · Alternativer Lebensstil}

\section{Zusammenfassung}

Zwei kürzlich nach Peer Review veröffentlichte Fachartikel zeigen ein vorteilhafteres Körpergewicht bei den Bevölkerungsgruppen i) der Nutzer von Komplementärmedizin in der Schweiz und ii) der Befürworter anthroposophischer Medizin und biologischer Ernährung in den Niederlanden. Diese Studien deuten somit darauf hin, dass die Nutzung komplementärer Formen der Medizin und/ oder assoziierte Lebensstile dem vorherrschenden Trend zu Übergewicht und Adipositas wenigstens teilweise entgegenwirken können. In der vorliegenden Übersichtsarbeit werden die Resultate beider Studien gegenübergestellt und die mögliche Einbindung der komplementären Medizin in einen präventiven Approach diskutiert.

(c) 2015 S. Karger GmbH, Freiburg

\section{Einleitung}

Trotz Fortschritten in der Prävention und im Management der Herz-Kreislauf-Erkrankungen bleiben diese weltweit eine Hauptursache frühzeitigen Todes. HerzKreislauf-Erkrankungen hängen nicht nur mit genetischen Veranlagungen zusammen, sondern sie werden sehr stark durch gut bekannte Risikofaktoren - ungünstige Ernährung, Stress, Bewegungsmangel, Rauchen, Alkoholkonsum und Umweltverschmutzung - beeinflusst. Im Rahmen von Public-Health-Massnahmen setzen die Behörden häufig auf Kampagnen gegen die erwartungsgemäss am ehesten steuerbaren Risikofaktoren, z.B. gegen Übergewicht oder - was damit häufig einhergeht - für mehr Bewegung. Diese Kampagnen beabsichtigen Anpassungen des persönlichen Lebensstils, erzielen aber langfristig nur eingeschränkt Ergebnisse. Weltweit ist Über-

\author{
Keywords \\ Obesity · Prevention - Use of complementary medicine \\ Organic diet · Alternative lifestyle
}

\section{Summary}

Can Complementary Medicine Make an Active Contribution to the Prevention of Obesity?

Two recent peer-reviewed publications show a more favourable body weight in the population groups i) of complementary medicine users in Switzerland and ii) of users of anthroposophic medicine and organic food in the Netherlands. Thus, these studies suggest that the use of complementary forms of medicine and/or associated lifestyles might at least in part counteract the prevailing trend towards overweight and obesity. In this review, the results of both studies are compared and the possible integration of complementary medicine in prevention is discussed.

๑c 2015 S. Karger GmbH, Freiburg

gewicht ein stetig wachsendes Gesundheitsproblem [1]. Ein Vergleich der Ergebnisse der Schweizerischen Gesundheitsbefragung (SGB) der Jahre 1992, 1997 und 2002 zeigt eine steigende Tendenz zu leichtem Übergewicht und Adipositas [2]. Auch wenn Übergewicht wahrscheinlich mit falscher Ernährung und/oder mangelnder Bewegung zusammenhängt, hat es vielfältigere Ursachen: Dazu gehören biologische Faktoren (Geschlecht, Alter, neuroendokrine Faktoren und genetische Veranlagung), Verhaltensfaktoren (beruhend auf soziopsychologischen Determinanten wie Gewohnheiten, Emotionen, Einstellungen, Glauben und persönlichen Erkenntnissen), Umweltfaktoren (bedingt durch das physische, wirtschaftliche und soziokulturelle Umfeld, in dem der Mensch lebt) und iatrogene Faktoren (durch die Einnahme gewisser Medikamente) [2]. Einige dieser Faktoren scheinen zwangsläufig mit Aspekten der Industriegesellschaften und dem dort

\section{KARGER \\ Fax +497614520714 Information@Karger.com} www.karger.com 
geförderten Lebensstil zusammenzuhängen. In diesen Gesellschaften können aber unterschiedliche Lebensstile nebeneinander koexistieren. Solche Lebensstile sind einerseits durch unterschiedliche praktische Entscheide gekennzeichnet - bezüglich Ernährung, körperlicher Aktivität, Rauchen, Alkoholkonsum, Bildung, Nutzung ausschliesslich konventioneller oder aber eher integrativer Medizin, Teilnahme am kulturellen Leben, sozialen Beziehungen usw. -, andererseits beruhen sie auf unterschiedlichen Ideologien und werden dadurch motiviert. Im vorliegenden Text wird der Lebensstil der Mehrheit der Menschen in Mitteleuropa als konventionell bezeichnet, und Lebensstile, die sich erkennbar davon unterscheiden (weil sie Alternativen zu ausschliesslich konventioneller Medizin, konventioneller Ernährung und/oder konventionellen Schulen suchen), werden als alternativ bezeichnet.

Der Einsatz von Komplementärmedizin in der Schweiz sowie ihre Wirksamkeit, Angemessenheit und Effizienz wurden 2005 durch das staatliche Programm zur Evaluation der Komplementärmedizin untersucht [3-5]. Dies zeigte, dass ungefähr die Hälfte der Bevölkerung irgendeine Form von Komplementärmedizin in Anspruch nahm. Die Popularität dieser Art von Medizin wurde untermauert durch eine Volksabstimmung im Jahr 2009, in der sich etwa zwei Drittel der Bürger für die Möglichkeit aussprachen, Komplementärmedizin zu nutzen. Für Einwohner der Schweiz deckt eine Gesundheitsgrundversicherung ein umfassendes Paket an Gesundheitsleistungen ab, zu dem von 1999 bis 2004 und nun wieder seit 2011 auch Therapien der Komplementärmedizin gehören, wenn sie von einem Arzt mit anerkannter KomplementärmedizinAusbildung verschrieben werden. Ausserdem entscheiden sich etwa 60\% der Einwohner für eine Zusatzversicherung, die unter anderem auch Zusatzausgaben im Zusammenhang mit Komplementärmedizin vergütet.

Zahlreiche Veröffentlichungen deuten darauf hin, dass sich Nutzer von Komplementärmedizin bezüglich soziodemographischer Charakteristika von Nichtnutzern unterscheiden: Weibliches Geschlecht, mittleres Alter und hoher Bildungsgrad sind bekannte Prädiktoren für die Nutzung von Komplementärmedizin (siehe z.B. [6-8]). Darüber hinaus gibt es aber Hinweise darauf, dass sich Nutzer und Nichtnutzer von komplementärer Medizin in mehreren gesundheitsrelevanten Merkmalen unterscheiden können. Nutzer komplementärer Medizin sind nach ihren eigenen Angaben insgesamt mehr krank als Nichtnutzer $[7,8]$. Dagegen zeigte ein früherer Bericht, dass Patienten, die anthroposophische Ärzte aufsuchen, seltener an Übergewicht leiden als jene, die zu konventionellen Ärzten gehen [9]. Auch weisen ehemalige Steiner-Schüler im Durchschnitt einen niedrigeren Body-Mass-Index (BMI) auf als eine zufällig gewählte vergleichbare Population [10]. Zwei vor Kurzem nach Peer Review veröffentlichte Fachartikel zeigen eine niedrigere Prävalenz von
Übergewicht bei den beiden Bevölkerungsgruppen, d.h. i) der Nutzer von Komplementärmedizin in der Schweiz [11] und ii) der Befürworter anthroposophischer Medizin und biologischer Ernährung in den Niederlanden [12]. Diese Studien deuten somit darauf hin, dass die Nutzung komplementärer Medizin und/oder assoziierte Lebensstile dem vorherrschenden Trend zu Übergewicht entgegenwirken, d.h., zur Prävention von Herz-KreislaufErkrankungen beitragen können (Abb. 1). Beide Arbeiten werden im Folgenden dargestellt, die verwendeten Methodologien erläutert und die erzielten Ergebnisse - insbesondere diejenigen zu Übergewicht - hervorgehoben (zusätzliche Informationen können den Originalpublikationen $[11,12]$ entnommen werden).

\section{Gesundheitliche Merkmale von Nutzern und Nichtnutzern von Komplementärmedizin: Methoden und Ergebnisse einer Schweizer Querschnittserhebung}

Landesweite Querschnittsbefragungen der Bevölkerung werden von der Schweizer Regierung alle 5 Jahre angeordnet und vom Bundesamt für Statistik durchgeführt (siehe [13]).Ziel jeder Befragung istes, Daten zu demographischen Merkmalen, zum Gesundheitsstatus und zur Nutzung von Gesundheitsdiensten sowie zu Lebensumständen und Charakteristika des Lebensstils der Befragten zu sammeln. Die letzten verfügbaren Daten wurden 2007 erhoben (Schweizerische Gesundheitsbefragung (SGB) 2007); hierzu wurde eine repräsentative Stichprobe von etwa 30000 Haushalten bestimmt. Die Zielpopulation der SGB2007 waren Einwohner der Schweiz im Alter von mindestens 15 Jahren, die einen Festnetzanschluss hatten und eine der drei Landessprachen Deutsch, Französisch oder Italienisch sprachen. Informationen wurden mit standardisierten Telefonbefragungen und mit von den Befragten selbst auszufüllenden Fragebögen ermittelt. Anhand der SGB2007-Daten wurden Nutzer und Nichtnutzer von Komplementärmedizin durch demographische Aspekte, Gewichtsstatus, wahrgenommenen Gesundheitszustand, Ernährung, Gebrauch verschiedener Medikamentengruppen, Rauchen, Alkoholkonsum und einige Parameter zum Lebensstil charakterisiert [11]. Auf die Nutzung von Komplementärmedizin, die primäre Variable der vorliegenden Analyse, bezieht sich die SGB2007 mit der Frage (Nr. 10 des Fragebogens) «Wie viele Male haben Sie in den letzten 12 Monaten eine der folgenden Therapien in Anspruch genommen?», wobei Angaben gemacht werden konnten zu a) Akupunktur, b) Homöopathie, c) Phytotherapie, d) Shiatsu/Fussreflexzonenmassage, e) autogenes Training/Hypnose, f) Neuraltherapie, g) traditionelle chinesische Medizin ohne Akupunktur, h) anthroposophische Medizin, i) Bio-Resonanz, j) indische Medizin/Ayurveda, k) Osteopathie, l) 


\begin{abstract}
Abb. 1. Nutzung von Komplementärmedizin und alternative Lebensstile gehen einher mit niedrigeren Prävalenzen von Übergewicht und Adipositas. a

Daten der Schweizerischen Gesundheitsbefragung 2007 [11]; b Daten der niederländischen KOALA-Geburts-Kohortenstudie [12].
\end{abstract}

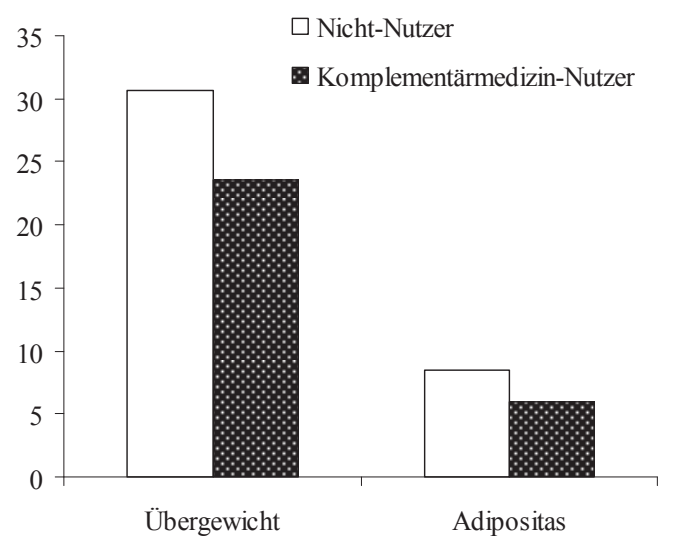

b

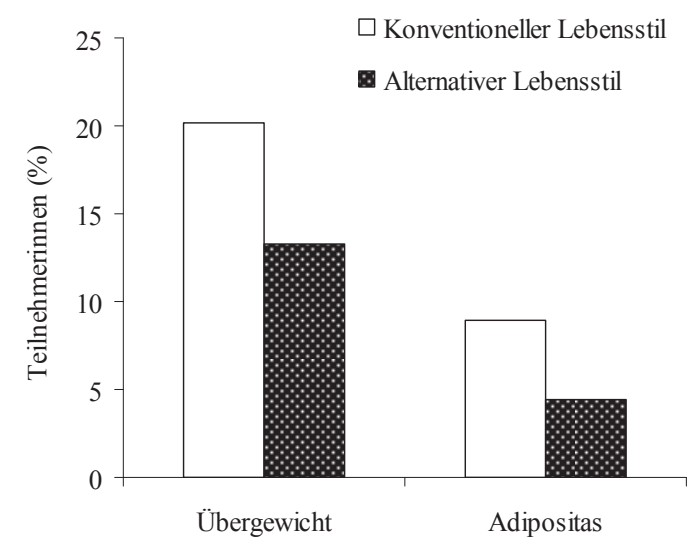

Sonstige, z.B. Kinesiologie, Feldenkrais-Methode usw. Jene Befragten, die irgendeine dieser Therapien mindestens einmal genutzt hatten, wurden als Nutzer von Komplementärmedizin angesehen und im vorliegenden Text so bezeichnet, die übrigen hingegen als «Nichtnutzer». Es wurden zahlreiche statistische Analysen durchgeführt, unter anderem univariate Analysen der KomplementärmedizinNutzung im Verhältnis zu jedem der potenziellen, anhand der SGB identifizierten Prädiktoren und multivariate Analysen der Komplementärmedizin-Nutzung im Verhältnis zu mehreren Gruppen dieser potenziellen Prädiktoren. In allen genannten Analysen wurde die erklärende Kraft der verschiedenen Prädiktoren mit multivariaten logistischen Regressionsmodellen ermittelt, in denen mittels eines Rückwärtseliminationsschemas anhand des Bayes'schen Informationskriteriums (BIC) eine Auswahl der zu behaltenden Prädiktoren getroffen wurde [14]. Alle Daten wurden unter Verwendung der Programmiersprache und Software-Umgebung $R$ analysiert [15].

Ungefähr ein Viertel aller Befragten hatte angegeben, mindestens einmal eine der aufgeführten komplementärmedizinischen Therapien genutzt zu haben (3333 von $14393 ; 23,2 \%$ ), während etwa zwei Drittel angegeben hatten, nichts davon genutzt zu haben (9821 von 14 393; $68,2 \%)$. Alle demographischen Variablen, nämlich Geschlecht, Alter, Landessprache, Nationalität, Einkommen, Haushaltsstruktur und Bildungsgrad, wurden in einem multivariaten logistischen Regressionsmodell berücksichtigt und erwiesen sich als unterschiedlich für Komplementärmedizin-Nutzer und Nichtnutzer. Die Ergebnisse zeigten, dass weibliches Geschlecht, jüngeres Alter, Französisch als Landessprache und höherer Bildungsgrad positive Indikatoren für die Nutzung von Komplementärmedizin waren. Danach wurden, zusätzlich zu den oben genannten demographischen Variablen, die verschiedenen krankheitsbezogenen Variablen in die multivariate logistische Regressionsanalyse integriert und die sich ergebenden Daten ebenfalls einer Rückwärtselimination, die auf dem BIC beruhte, unterzogen. Die Analyse zeigte deutlich, dass hohe BMI-Werte mit einer geringeren Wahrscheinlichkeit der Komplementärmedizin-Nutzung einhergingen - wohlbemerkt in Modellen, die auch die demographischen Merkmale berücksichtigten.

Weiter waren die Befragten gebeten worden, ihren subjektiv wahrgenommenen gegenwärtigen Gesundheitszustand qualitativ zu klassifizieren, allgemein anzugeben, ob sie chronische Krankheiten hätten, und zu spezifizieren, ob sie (zum Zeitpunkt der Befragung oder während der zurückliegenden 12 Monate) wegen mehrerer Krankheiten in medizinischer Behandlung gewesen waren. Zwar glaubte in allen Gruppen die Mehrheit der Befragten, bei guter Gesundheit zu sein, aber wahrgenommene schlechte oder sehr schlechte Gesundheit war bei Komplementärmedizin-Nutzern etwas häufiger vertreten als bei Nichtnutzern. Weiter verwendeten Komplementärmedizin-Nutzer signifikant weniger Medikamente gegen hohen Blutdruck und hohes Cholesterin und tendenziell weniger Herz- und Diabetes-Medikamente. Schliesslich wurde in der SGB2007 nach mehreren Parametern des Lebensstils gefragt, z.B. Ernährung, wahrgenommene körperliche Aktivität, Berücksichtigung gesundheitlicher Belange und Sicherheit bezüglich gesundheitlicher Themen. Komplementärmedizin-Nutzer assen tendenziell öfter Gemüse und seltener Fleisch und waren sich weniger oft sicher als Nichtnutzer, genügend körperlich aktiv zu sein. Interessanterweise hatten KomplementärmedizinNutzer öfter den Eindruck, ihre Überlegungen zu Gesundheit beeinflussten ihren Lebensstil, und waren sich häufiger sicher über ihr Gesundheitsverhalten.

\section{Einfluss alternativer Lebensstile auf das Körpergewicht bei Frauen: Methoden und Ergebnisse}

Die niederländische KOALA-Geburtskohortenstudie ist eine prospektive Studie mit 2834 Mutter-Kleinkind-Paaren. KOALA ist ein (niederländisches) Akronym für «Kind, 
Ouders en gezondheid: Aandacht voor Leefstijl en Aanleg» (Kinder, Eltern und Gesundheit: ein Blick auf Lebensstil und Konstitution). In Hebammenpraxen der südlichen Niederlande wurden gesunde schwangere Frauen aus einer anderen prospektiven Kohortenstudie rekrutiert [16]. Um Frauen mit verschiedenen Lebensstilen in die Studie einzubeziehen, wurden über mehrere zusätzliche, spezifische Kanäle schwangere Frauen rekrutiert, etwa über Läden für Bio-Lebensmittel, anthroposophische Allgemeinmediziner und Hebammen, anthroposophische Kliniken für Kinder unter 5 Jahren, Rudolf-Steiner-Schulen und themenbezogene Zeitschriften. Ziel der vor Kurzem veröffentlichten Auswertung [12] war die Beschreibung der mit alternativen Lebensweisen zusammenhängenden Merkmale weiblicher Gesundheit während der Mutterschaftsperiode, mit Schwerpunkt auf dem Gewichtsstatus, und zwar anhand des Vergleichs der zwei verschiedenen Rekrutierungsgruppen der KOALA-Studie, d.h. einer Gruppe mit konventionellem und einer anderen Gruppe mit sehr wahrscheinlich alternativem Lebensstil.

Alle Frauen wurden zwischen ihrer 14. und 18. Schwangerschaftswoche rekrutiert und bekamen detaillierte Fragebogen zu soziodemographischen und gesundheitlichen Merkmalen wie auch zu persönlichen Gewohnheiten und Ernährung zugeschickt. Als ihre Kinder 4-5 Jahre alt waren, erhielten die Frauen schliesslich einen Fragebogen, der Fragen zu ihrem Gewicht enthielt. Die Analyse stützte sich auf die Daten zu 2333 von 2343 Müttern in der konventionellen Gruppe und 485 von 491 in der alternativen Gruppe. Hauptdeterminante (Exponierung) war der alternative Lebensstil in der alternativen Gruppe, der mit dem konventionellen Lebensstil in der konventionellen Gruppe verglichen wurde. Unterschiede zwischen den beiden Rekrutierungsgruppen wurden evaluiert mit ANOVA oder Chi-Quadrat-Test für kontinuierliche bzw. kategorische Variablen. Die lineare Regressionsanalyse wurde mit dem BMI (vor Schwangerschaft und 4-5 Jahre nach Entbindung) als kontinuierlicher Outcome-Variable und mit der Rekrutierungsgruppe als unabhängiger Variable versehen. Mögliche Einflüsse von ConfounderVariablen der Rekrutierungsgruppen wurden eingeschätzt, indem das Alter der Mutter bei Entbindung, die Zahl der vorherigen Kinder und die geographische Region den Regressionsmodellen hinzugefügt wurden. Ebenso wurde logistische Regression verwendet, um die Odds Ratio für Übergewicht und Adipositas beim Vergleich der alternativen und der konventionellen Gruppe abzuschätzen, mit und ohne Anpassung wegen möglicher Confounder-Variablen. Alle Daten wurden mit IBM ${ }^{\circledR}$ SPSS $^{\circledR}$ Statistics 19 analysiert.

Mütter der alternativen Gruppe hatten, verglichen mit jenen der konventionellen Gruppe, häufiger eine höhere Bildung, waren bei der Entbindung durchschnittlich 2 Jahre älter und hatten häufiger schon vorher mindestens ein Kind geboren. Bezüglich der Wohnorte unterschieden sich die beiden Gruppen: Die meisten Frauen mit alternativen Lebensstilen lebten in den mittleren nördlichen Provinzen, die meisten Frauen mit konventionellem Lebensstil hingegen lebten in südlichen Provinzen (Limburg und Brabant). Wie aufgrund der Art der Rekrutierung zu erwarten war, hielten sich mehr Frauen aus der alternativen Gruppe an bestimmte Lebensregeln - meistens an Regeln des Vegetarismus und der Anthroposophie - und wählten biologische und biodynamische Produkte. Der Prozentsatz der Frauen, die in der 34. Schwangerschaftswoche aktiv rauchten, war in der alternativen Gruppe deutlich geringer als in der konventionellen Gruppe, und es waren in der ersteren Gruppe auch weniger Frauen passivem Rauchen ausgesetzt. Im Gegensatz dazu wurde in der alternativen Gruppe etwas mehr Alkohol während der Schwangerschaft konsumiert als in der konventionellen Gruppe. In der alternativen Gruppe gaben weniger Frauen Bluthochdruck während der Schwangerschaft an als in der konventionellen Gruppe. Allgemein war die $\mathrm{Zu}$ friedenheit mit dem Leben nach den Angaben der Mütter hoch, aber dennoch war der Prozentsatz der Zufriedenen in der alternativen Gruppe niedriger als in der konventionellen Gruppe. Auch der allgemeine Gesundheitszustand wurde in der alternativen Gruppe als etwas schlechter eingestuft.

Der durchschnittliche BMI war bei Beginn der Schwangerschaft in der alternativen Gruppe leicht, aber signifikant niedriger als in der konventionellen Gruppe. Ein vergleichbarer Unterschied wurde 4-5 Jahre nach der Entbindung beobachtet. Bezüglich Gewichtszunahme während der Schwangerschaft wurden keine Unterschiede festgestellt, jedoch war die gesamte Gewichtszunahme zwischen der Schwangerschaft und 4-5 Jahre nach der Entbindung in der alternativen Gruppe leicht höher, obgleich die BMI-Werte niedriger waren - nicht nur zum ersten, sondern auch zum späteren Zeitpunkt. Zwar wies die Mehrheit der Frauen in beiden Rekrutierungsgruppen einen BMI im normalen Bereich auf, aber die Verteilung der Teilnehmerinnen auf die verschiedenen BMI-Kategorien unterschied sich für die beiden Gruppen signifikant, sowohl zu Beginn der Schwangerschaft als auch 4-5 Jahre nach der Entbindung. Ein normaler BMI kam in der alternativen Gruppe häufiger vor als in der konventionellen Gruppe, sowohl zu Beginn der Schwangerschaft als auch 4-5 Jahre nach der Entbindung. In der konventionellen Gruppe kam Untergewicht zu beiden Zeitpunkten seltener vor, Übergewicht und Adipositas hingegen beträchtlich häufiger.

Da sich die Mütter beider Gruppen bezüglich ihres Alters, der Zahl vorheriger Kinder und des Wohnortes unterschieden und erwartet wurde, dass sich diese Faktoren auf den BMI auswirken könnten, wurde eine multiple lineare Regressionsanalyse durchgeführt, mit dem 
BMI (vor der Schwangerschaft und 4-5 Jahre nach der Entbindung) als abhängiger Variable und der Rekrutierungsgruppe als der hauptsächlichen unabhängigen $\mathrm{Va}$ riablen. Die anderen erwähnten Faktoren wurden zu dem Modell hinzugenommen, um potenzielle Confounders zu korrigieren. Nach der Korrektur wegen potenzieller Confounders war der Regressionskoeffizient abgeschwächt, blieb aber immer noch statistisch signifikant. Auch das Risiko, vor der Schwangerschaft übergewichtig oder dick zu sein, war in der alternativen Gruppe niedriger als in der konventionellen. Ähnliche Resultate ergaben sich für den BMI 4-5 Jahre nach der Entbindung.

\section{Einschränkungen der analysierten Studien}

Beide Studien hatten einen explorativen Charakter, und die erzielten Ergebnisse müssen deswegen mit Vorsicht interpretiert werden. Insbesondere kann eine genaue kausale Beziehung zwischen Nutzung von Komplementärmedizin und niedriger Prävalenz von Übergewicht nicht bewiesen werden, da zahlreiche Faktoren bei den beobachteten Zusammenhängen eine Rolle spielen können. Gerade der Einfluss der sozioökonomischen Merkmale ist nicht zu vernachlässigen, da sie bekannterweise die Prävalenz von Übergewicht beeinflussen können. Es sei aber darauf hingewiesen, dass in der SGB2007-Auswertung der Effekt des Einkommens - das häufig die sozioökonomischen Merkmale weitgehend bestimmt - statistisch ausgeschaltet werden konnte. Auch in der KOALA-Auswertung wurden mehrere Faktoren in der Analyse berücksichtigt, die mit sozioökonomischen Merkmalen korrelieren können (Frauenalter, Zahl vorheriger Kinder und Wohngegend). In einer Unterauswertung ausschliesslich der Teilnehmerinnen mit einem hohen Ausbildungsgrad blieb der Unterschied zwischen beiden Rekrutierungsgruppen erhalten (Simões-Wüst et al., unveröffentlichte Daten).

Eine weitere Limitierung der vorliegenden Arbeit liegt darin, dass die Daten von den Teilnehmerinnen selbst angegeben wurden. Insbesondere könnten die Angaben zum Gewicht für die vorliegende Arbeit kritisch sein. Die Genauigkeit und Zuverlässigkeit eigener Angaben zu Gewicht und Grösse sind aber, wie gezeigt wurde, annehmbar; Personen mit einem BMI im normalen Bereich (wie die Mehrheit der Teilnehmenden an beiden Erhebungen) machen sehr genaue Angaben, Personen mit Übergewicht sind anfälliger dafür, ihr Gewicht zu niedrig anzugeben, untergewichtige Personen hingegen zu hoch [17]. In der vorliegenden Studie hätten aber solche tendenziellen Fehlangaben zu einer Unterschätzung der Unterschiede zwischen den verglichenen Gruppen geführt, nicht zu einer Überschätzung - dies unterstützt also die Validität der gefundenen Unterschiede.
Schliesslich muss bei einer Gegenüberstellung beider Studien berücksichtigt werden, dass unterschiedliche Gruppen verglichen wurden. Während in der SGB2007 die Nutzung irgendeiner Therapie der Komplementärmedizin für die Gruppeneinteilung massgebend war, fand die Gruppenzuordnung in der Auswertung der KOALADaten anhand der Rekrutierungsart statt. In der KOALAStudie wurden die Frauen der «alternativen Gruppe» aktiv über mehrere spezifische Kanäle rekrutiert (Läden für Bio-Lebensmittel, anthroposophische Allgemeinmediziner und Hebammen, anthroposophische Kliniken für Kinder unter 5 Jahren, Rudolf-Steiner-Schulen und themenbezogene Zeitschriften) und mit Frauen verglichen, die ohne irgendeine lebensstilbasierte Selektion rekrutiert wurden. Und schliesslich wurde die Intensität der Nutzung von Komplementärmedizin in keiner der beiden Studien berücksichtigt, was zu einer Unterschätzung des Potenzials der Nutzung von Komplementärmedizin geführt haben könnte.

\section{Nutzung komplementärer Medizin und Behandlung von Herz-Kreislauf-Erkrankungen}

Übergewicht ist ein wichtiger Risikofaktor für HerzKreislauf-Erkrankungen. Schon heute sollten in der konventionellen Medizin therapeutische Veränderungen des Lebensstils - mehr körperliche Aktivität und gesündere Ernährung; Aufgabe des Rauchens - die ersten Ziele einer Standardstrategie zur Verringerung kardiovaskulärer Risiken sein [1, 18]. Die SGB2007-Auswertung [11] zeigt, dass Komplementärmedizin-Nutzer weniger Medikamente gegen kardiovaskuläre Erkrankungen nahmen, d.h. gegen hohen Blutdruck, hohe Cholesterinwerte und Herzprobleme (und per Trend Diabetes); dies auch nach Kompensation der Einwirkungen von demographischen Faktoren, wahrgenommenem Gesundheitszustand und, interessanterweise, BMI. Letzteres bedeutet, dass der geringe Verbrauch solcher Medikamente nicht einfach durch eine geringere ärztliche Einschätzung ihrer Notwendigkeit anhand des niedrigen BMI erklärt werden kann. Es müssen andere, eventuell mit dem Lebensstil verbundene Gründe sein, die zu einer eher konservativen Behandlung der entsprechenden Erkrankungen geführt haben. Bei der Auswertung wurden keine Hinweise auf negative Auswirkungen einer möglichen Unterversorgung gefunden, und eine frühere Arbeit zum Langzeitüberleben zeigte sogar, dass Patientinnen und Patienten, deren Hausarzt eine zusätzliche Ausbildung in Komplementärmedizin hatte, länger leben [19]. Daten aus dem Schweizer Programm zur Evaluation der Komplementärmedizin ergaben, dass Komplementärmediziner bei der Behandlung von Herz-KreislaufErkrankungen (am häufigsten Bluthochdruck, chronische ischämische Herzkrankheit, nicht näher bezeichnete 
Herzinsuffizienz, Vorhofflimmern und -flattern) zwar meistens auf konventionelle Therapien setzen [20], jedoch wurden 13\% der Patientinnen und Patienten ausschliesslich mit Therapien der Komplementärmedizin behandelt. Die Folgen eines reduzierten Verbrauchs von Medikamenten gegen Herz-Kreislauf-Erkrankungen durch einige Komplementärmedizin-Nutzer sollten in speziell für diesen Zweck konzipierten Studien untersucht werden. Es wäre interessant zu erfahren, was genau die verschiedenen Formen der Komplementärmedizin für das Management von Herz-Kreislauf-Erkrankungen anbieten und wie Komplementärmediziner das individuelle Risiko für Herz-Kreislauf-Erkrankungen beurteilen. Die aktive Beteiligung von Komplementärmedizinern an der noch andauernden Diskussion darüber, welche Patientinnen und Patienten mit Medikamenten gegen zu hohen Cholesterinspiegel [18] und hohen Blutdruck [21] behandelt werden sollten, erscheint dringend. Medikamente gegen Herz-Kreislauf-Erkrankungen müssen täglich eingenommen werden, oft von der Diagnose an das ganze Leben lang, und gehen mit gut bekannten Nebenwirkungen einher (siehe z.B. $[18,22]$ ).

\section{Gesundheitsökonomische Überlegungen}

Gesundheitsökonomisch könnten sowohl der oben genannte geringere Verbrauch von Medikamenten gegen Herz-Kreislauf-Erkrankungen als auch die geringere Prävalenz von Übergewicht bei KomplementärmedizinNutzern interessant sein. In der Schweiz sind Medikamente gegen Herz-Kreislauf-Erkrankungen für 14,1\% der Kosten gemäss Fabrikabgabepreisen verantwortlich [23], Medikamente gegen hohen Blutdruck und hohe Cholesterinwerte entsprechen $15 \%$ der gesamten Medikamentenkosten [24], und 8 der 25 am meisten verkauften Medikamente wirken gegen hohen Blutdruck oder hohe Cholesterinwerte [25]. Weiter liegen die indirekten und direkten Kosten von Übergewicht pro Kopf und Jahr bei 1600 CHF und diejenigen von Adipositas bei 5800 CHF [26]. Die zwei hier dargestellten Studien liefern zahlreiche Hinweise darauf, dass Komplementärmedizin-Nutzer mehr eigene finanzielle Ressourcen in die Gesundheitserhaltung investieren und somit die allgemeinen Gesundheitsausgaben entlasten.

\section{Zusammenhang zwischen Nutzung komplementärer Medizin und niedriger Prävalenz von Übergewicht}

Die oben beschriebenen Arbeiten deuten auf einen vielfach vorher vermuteten - Zusammenhang zwischen einerseits Nutzung von Komplementärmedizin und/oder
Entscheidung für einen alternativen Lebensstil, andererseits niedriger Prävalenz von Übergewicht hin (Abb. 1). Die Vorgehensweise beider ausgewerteten Studien legt nahe und die gewonnenen Ergebnisse bestätigen, dass tatsächlich eine für die Mehrheit der Bevölkerung repräsentative Gruppe verglichen wurde mit einer Gruppe, die eher Komplementärmedizin nutzt und einen alternativen Lebensstil pflegt.

Es ist gegenwärtig nicht möglich, genau anzugeben, welche Faktoren der Nutzung komplementärer Medizin oder eines alternativen Lebensstils für die beobachteten Unterschiede bei der Prävalenz von Übergewicht verantwortlich sind. Anhand der Popularität der Komplementärmedizin in der Schweiz und der Dringlichkeit der Mobilisierung der Bevölkerung für die Bekämpfung der Übergewichtsproblematik ist eine entsprechende Diskussion äusserst wünschenswert. Da Übergewicht sehr wahrscheinlich durch ein Ungleichgewicht in Ernährung und Energieverbrauch entsteht, darf angenommen werden, dass Nutzer komplementärer Medizin eine niedrigere Energieaufnahme und/oder einen höheren Energieverbrauch aufweisen. Letzteres würde auf häufigere bewegungsreiche bzw. seltenere sitzende Aktivitäten hindeuten. Die Teilnehmerinnen der KOALA-Studie mit alternativen Lebensstilen ernährten sich häufiger vegetarisch, und Befragte der SGB2007 assen tendenziell öfter Gemüse und seltener Fleisch. Vegetarische Ernährung geht einher mit einem geringeren Risiko bezüglich mehrerer chronischer Krankheiten, was sich an niedrigeren Konzentrationen von Lipoproteinen geringer Dichte, niedrigerem Blutdruck sowie niedrigeren Raten von Bluthochdruck und Diabetes vom Typ 2 zeigt [27]. Es ist zudem bekannt, dass Vegetarismus zu einem normalen Gewicht beitragen kann [28, 29]. Bei Erwachsenen mit zu hohem Blutdruck kann darüber hinaus das Essen von Früchten und Gemüse das lipidbedingte kardiovaskuläre Risiko mässigen [30]. Eine absolut höhere Aufnahme von Milchprodukten - die häufig als Alternative zu Fleisch verzehrt werden - kann die Risiken von Übergewicht, Adipositas [31] und metabolischem Syndrom [32] verringern.

Nicht nur die Zusammensetzung der Nahrung, sondern auch die Herkunft der Lebensmittel kann eine Rolle spielen. Die Teilnehmerinnen der KOALA-Studie mit alternativen Lebensstilen verzehrten viel öfter biologische Lebensmittel, die zum Teil andere Eigenschaften aufweisen als konventionell produzierte Lebensmittel. Beispielsweise enthalten Kuhmilch und Wiederkäuerfleisch von Tieren, die nach den Richtlinien biologischer Produktion gehalten [33,34] oder mit Gras gefüttert werden [35, 36], wie das bei biologischer Landwirtschaft oft der Fall ist, höhere Konzentrationen an konjugierter Linolsäure als konventionelle Milch. Es wurde bislang angenommen, dass sich konjugierte Linolsäure positiv auf das Gewicht auswirkt; eine neuere Übersichtsarbeit ergab jedoch in- 
konsistente Ergebnisse [37]. Diese Übersichtsarbeit basierte auf kontrollierten Studien über die Wirkung einer Nahrungsergänzung mit konjugierter Linolsäure auf den Metabolismus und das Gewicht bei Menschen. Eventuell sind die unterschiedlichen Ergebnisse auf verschiedene Dosierung, Zusammensetzung und Quellen (synthetische vs. natürliche) zurückzuführen.

Die Entscheidung für biologische Lebensmittel kann auch die Produktwahl beeinflussen: Eine Umfrage unter Konsumenten in den Niederlanden [38] zeigte, dass der Konsum biologischer Lebensmittel oft mit der Verwendung von frischer zubereiteten Lebensmitteln und weniger Fertigmahlzeiten einhergeht. Darüber hinaus liess sich für die Untergruppe jener an der KOALA-Studie teilnehmenden Mütter, die eine Milchprobe abgaben, zeigen, dass ein höherer Konsum von Fleisch- und Milchprodukten aus biologischer Landwirtschaft in der alternativen Gruppe mit einer geringeren Aufnahme partiell hydrierter (gehärteter) Fette und Öle in industriell hergestellten Lebensmitteln (gemessen anhand des Quotienten von Vaccensäure zu Elaidinsäure in der Muttermilch [39]) einherging. Die Frauen in der alternativen Gruppe haben demnach anscheinend weniger jener Produkte - häufig hochkalorisch, zum Naschen geeignet - verzehrt, die partiell hydrierte (gehärtete) Fette und Öle enthalten. Eine Meta-Analyse von Kohortenstudien deutet übrigens auf eine schädigende Wirkung partiell hydrierter (gehärteter) Fette und Öle bezüglich koronarer Herzkrankheit hin [40].

Die geringere Prävalenz von Übergewicht bei Nutzern von Komplementärmedizin könnte neben den direkten Ursachen - Ernährung und Energieverbrauch - auch damit zusammenhängen, dass die Nutzung von Komplementärmedizin mit anderen Gewohnheiten und vor allem Einstellungen einhergeht. Ein Aspekt könnte ein gewisser Verzicht sein, der sich in weniger Rauchen und moderatem Alkoholkonsum ausdrücken könnte. Die Daten der SGB2007 deuten jedenfalls tendenziell auf selteneres Rauchen und niedrigeren Alkoholkonsum bei Komplementärmedizin-Nutzern hin (Simões-Wüst et al., unveröffentlichte Daten). Auch die Auswertung der KOALA-Daten zeigt, dass die Frauen der alternativen Gruppe seltener aktiv rauchten und seltener passivem Rauchen ausgesetzt waren.

Sowohl in der SGB2007-Erhebung als auch in der KOALA-Auswertung schienen sich die Teilnehmer der alternativen Gruppe (Komplementärmedizin-Nutzer bzw. Frauen mit alternativen Lebensstilen) durch eine besonders sorgfältige Gestaltung ihres gesundheitsrelevanten Verhaltens von der allgemeinen Bevölkerung abzuheben. In der SGB2007 hatten Komplementärmedizin-Nutzer öfter den Eindruck, ihre Überlegungen zu Gesundheit beeinflussten ihren Lebensstil, und waren sich häufiger sicher über ihr Gesundheitsverhalten; die Teilnehmerin- nen der KOALA-Studie mit alternativen Lebensstilen hielten sich häufiger an Lebensregeln. Die ursprüngliche Motivation, die bei der gesundheitsfördernden Gestaltung des Verhaltens hilfreich ist, verdient weitere Forschung; bisher nicht beachtete Aspekte wie Willenskraft, Achtsamkeit und Spiritualität könnten dabei eine nicht zu vernachlässigende Rolle spielen.

\section{Möglicher Beitrag der Komplementärmedizin zur Prävention von Übergewicht}

Die vorliegende Übersichtsarbeit unterstreicht die Notwendigkeit, die verschiedenen Lebensstile, die in unserer pluralistischen Gesellschaft existieren, unter die Lupe zu nehmen und aus ihren Errungenschaften zu lernen. Während die Public-Health-Experten sich darüber austauschen, wie eine Kampagne gegen Übergewicht zu gestalten ist, und Möglichkeiten wie finanzielle Anreize zum Abnehmen abwägen [41], zeigen die hier erwähnten Publikationen [11, 12], dass es Bevölkerungsgruppen gibt, die schon jetzt durch ihre persönlichen Entscheidungen mögliche Wirkungen solcher präventiven Massnahmen übertreffen. Dass es sich dabei eher um eine «Bottom up»-Bewegung handelt, wie übrigens bei der Nutzung der Komplementärmedizin per se, ist naheliegend. Diese Bevölkerungsgruppen scheinen ihre Gesundheit besonders ernst zu nehmen und dazu bereit zu sein, ihren Lebensstil wenigstens teilweise gemäss gesundheitlicher Überlegungen zu gestalten. Diese Bereitschaft ist freiwillig, zeugt von persönlichem Engagement und scheint durch persönliche Einstellungen motiviert zu sein. Es sollte die Frage gestellt werden, wie mehr Menschen dazu verholfen werden kann, eine solche Bereitschaft zu entwickeln. Auch wenn die hier erwähnten Arbeiten keinen kausalen Zusammenhang zwischen Nutzung von Komplementärmedizin und seltenerem Vorkommen von Übergewicht beweisen können, deuten sie auf eine Korrelation zwischen beiden hin. Gerade in der Schweiz, wo sich die Komplementärmedizin einer grossen Beliebtheit erfreut, erscheint es erstrebenswert, dass ihr Potenzial bei der Prävention von Übergewicht vermehrt wahrgenommen wird. Es ist vorstellbar, dass viele der zahlreichen Komplementärmedizin-Sympathisanten noch nicht in der Lage sind, ihre eigenen gesundheitserhaltenden Ressourcen (vollständig) einzusetzen. Die Komplementärmedizin mit der dazugehörigen ganzheitlichen und individuellen Patientenwahrnehmung, Lebensstilberatung und Auswahl an zahlreichen gesundheitsfördernden Therapien könnte einen aktive(re)n Beitrag zur Prävention von Übergewicht leisten. Dass in diesem Zusammenhang die öffentliche Gesundheitsförderung von einem Dialog mit der Komplementärmedizin profitieren könnte, ist wahrscheinlich. 


\section{Dank}

Dr. med. Carel Thijs (Universität Maastricht, Niederlande), Dr. med. Christian Schikarski (Paracelsus-Spital Richterswil, Schweiz) und Dr. med. Boris Müller-Hübenthal (Paracelsus-Spital Richterswil, Schweiz) seien herzlich bedankt für sehr interessante wissenschaftliche Diskussionen. Für finanzielle Unterstützung dieser Übersichtsarbeit danke ich der ChristophorusStiftung (Stuttgart, Deutschland) und dem Trägerverein Paracelsus-Spital Richterswil (Richterswil, Schweiz).

\section{Disclosure Statement}

Die Autorin arbeitet in einem Spital mit Schwerpunkt Anthroposophische Medizin.

\section{Literatur}

1 Jackson E, Rubenfire M: Obesity, weight reduction, and cardiovascular disease. UpToDate 2012. www.uptodate.com/contents/ obesity-weight-reduction-and-cardiovasculardisease (aufgerufen am 29.05.2012).

2 Jordan MD, Kuntsche S, Gmel G: Übergewicht bei Erwachsenen in der Schweiz: Aspekte einer multifaktoriellen Problematik, Ergebnisse der Schweizerischen Gesundheitsbefragung 2002. Neuchâtel, Schweizerische Fachstelle für Alkohol- und andere Drogenprobleme, Bundesamt für Statistik, 2007.

$>3$ Walach H, Heusser P: Effektiv oder nicht? Entscheiden Sie selbst! - Die PEK-HTABerichte. Forsch Komplementmed 2006;13 (suppl 2):2-3.

4 Melchart D, Mitscherlich F, Amiet M, Eichenberger R, Koch P: Programm Evaluation Komplementärmedizin (PEK) - Schlussbericht. 24.04.2005. www.bag.admin.ch/themen/ krankenversicherung/00263/00264/04102/ index.html?lang\%20=\%20de.

$\checkmark 5$ Wolf U, Maxion-Bergemann S, Bornhöft G, Matthiessen PF, Wolf M: Use of complementary medicine in Switzerland. Forsch Komplementmed 2006;13(suppl 2):4-6.

6 Menniti-Ippolito F, Bologna E, Gargiulo L, Forcella E, Sabbadini LL, Raschetti R: Characteristics of unconventional medicine users and their households in Italy (article in Italian). Ann Ist Super Sanita 2004;40:455-461.

7 Bucker B, Groenewold M, Schoefer Y, Schäfer $\mathrm{T}$ : The use of complementary alternative medicine (CAM) in 1,001 German adults: results of a population-based telephone survey. Gesundheitswesen 2008;70:e29-36.

$>8$ Hanssen B, Grimsgaard S, Launsø L, Fønnebø V, Falkenberg T, Rasmussen NK: Use of complementary and alternative medicine in the Scandinavian countries. Scand J Prim Health Care 2005;23:57-62.

$\checkmark$ Unkelbach R, Abholz HH: Unterschiede zwischen Patienten schulmedizinischer und anthroposophischer Hausärzte. Forsch Komplementmed 2006;13:349-355.

$\checkmark 10$ Fischer HF, Binting S, Bockelbrink A, Heusser P, Hueck C, Keil T, Roll S, Witt C: The effect of attending Steiner schools during childhood on health in adulthood: a multicentre cross-sectional study. PLoS One 2013;8:e73135.

$>11$ Simões-Wüst AP, Rist L, Dettling M: Self-reported health characteristics and medication consumption by CAM users and nonusers: a Swiss cross-sectional survey. J Altern Complement Med 2014;20:40-47.
2 Simões-Wüst AP, Kummeling I, Mommers M, Huber MA, Rist L, van de Vijver LP, Dagnelie PC, Thijs C: Influence of alternative lifestyles on self-reported body weight and health characteristics in women. Eur J Public Health 2014;24:321-327.

13 Bundesamt für Statistik: Schweizerische Gesundheitsbefragung 2007: Konzept, Methode, Durchführung. $w w w . b f s . a d m i n . c h / b f s /$ portal/ de/index/infothek/erhebungen_quellen/blank/ blank/ess/03.html (aufgerufen am 25.05.2012).

14 Schwarz G: Estimating the dimension of a model. Ann Statist 1978;6:461-464.

15 R Development Core Team: A language and environment for statistical computing. Vienna, R Foundation for Statistical Computing, 2011.

16 Kummeling I, Thijs C, Penders J, Snijders BE, Stelma F, Reimerink J, Koopmans M, Dagnelie PC, Huber M, Jansen MC, de Bie R, van den Brandt PA: Etiology of atopy in infancy: the KOALA Birth Cohort Study. Pediatr Allergy Immunol 2005;16:679-684.

17 Lin CJ, DeRoo LA, Jacobs SR, Sandler DP: Accuracy and reliability of self-reported weight and height in the Sister Study. Public Health Nutr 2012;15:989-999.

18 Lewis SJ: Lipid-lowering therapy: who can benefit? Vasc Health Risk Manag 2011;7 525-534.

19 Kooreman P, Baars EW: Patients whose GP knows complementary medicine tend to have lower costs and live longer. Eur J Health Econ 2012;13:769-776.

20 Matter-Walstra K, Schoeni-Affolter F, Widmer M, Busato A: Patient-based evaluations of primary care for cardiovascular diseases: a comparison between conventional and complementary medicine. J Eval Clin Pract 2008; 14:75-82.

21 Mann JFE, Hilgers KF: Hypertension: who should be treated? UpToDate 2014. $w w w$. uptodate.com/contents/hypertension-whoshould-be-treated.

22 Alberton M, Wu P, Druyts E, Briel M, Mills EJ: Adverse events associated with individual statin treatments for cardiovascular disease: an indirect comparison meta-analysis. QJM 2012;105:145-157.

23 Interpharma: Marktanteile von Medikamenten nach Indikationsgebiet 2010. $w w w$. interpharma.ch/de (aufgerufen am 24.02.2012).

24 Schweizerisches Gesundheitsobservatorium: Obsan Rapport 50. www.obsan.admin.ch/bfs/ obsan/de/index/05/publikationsdatenbank. html?publicationID\%20\%204550 (aufgerufen am 25.05.2012).
25 Bundesrat: Medikamenten-Preisvergleich mit den Nachbarländern. www.parlament.ch/d/ dokumentation/curia-vista/vorstoesse-tabellengrafiken/Documents/cv-08-3187-d.pdf (aufgerufen am 18.02.2012).

26 Schneider H, Venetz W, Berardo CG: Overweight and obesity in Switzerland, Part 1: cost burden of adult obesity in 2007. Bericht im Auftrag des BAG. Basel, HealthEcon, 2009.

27 Craig WJ, Mangels AR: Position of the American Dietetic Association: vegetarian diets. J Am Diet Assoc 2009;109:1266-1282.

28 Craig WJ: Nutrition concerns and health effects of vegetarian diets. Nutr Clin Pract 2010;25:613-620.

29 Sticher MA, Smith CB, Davidson S: Reducing heart disease through the vegetarian diet using primary prevention. J Am Acad Nurse Pract 2010;22:134-139.

30 Adebawo O, Salau B, Ezima E, Oyefuga O, Ajani E, Idowu G, Famodu A, Osilesi O: Fruits and vegetables moderate lipid cardiovascular risk factor in hypertensive patients. Lipids Health Dis 2006;5:14.

31 Louie JC, Flood VM, Hector DJ, Rangan AM, Gill TP: Dairy consumption and overweight and obesity: a systematic review of prospective cohort studies. Obes Rev 2011;12:e582-592.

32 Crichton GE, Elias MF, Buckley JD, Murphy KJ, Bryan J, Frisardi V: Metabolic syndrome, cognitive performance, and dementia. J Alzheimers Dis 2012;30(suppl 2):S77-87.

33 Jahreis G, Fritsche J, Steinhart H: Conjugated linoleic acid in milk fat: high variation depending on production system. Nutr Res 1997;17:1479-1484.

34 Bergamo P, Fedele E, Iannibelli L, Marzillo G: Fat-soluble vitamin contents and fatty acid composition in organic and conventional Italian dairy products. Food Chemistry 2003;82:625-631.

35 Jutzeler van Wijlen RP, Colombani PC: Grass-based ruminant production methods and human bioconversion of vaccenic acid with estimations of maximal dietary intake of conjugated linoleic acids. Int Dairy J 2010; 20:433-448.

36 Dannenberger D, Nuernberg G, Scollan N, Schabbel W, Steinhart H, Ender K, Nuernberg K: Effect of diet on the deposition of $n-3$ fatty acids, conjugated linoleic and C18:1trans fatty acid isomers in muscle lipids of German Holstein bulls. J Agric Food Chem 2004;52:6607-6615. 
37 McCrorie TA, Keaveney EM, Wallace JM, Binns N, Livingstone MB: Human health effects of conjugated linoleic acid from milk and supplements. Nutr Res Rev 2011;24:206-227.

38 van de Vijver LP, van Vliet ME: Health effects of an organic diet-consumer experiences in the Netherlands. J Sci Food Agric 2012;92:2923-2927.
9 Mueller A, Thijs C, Rist L, Simões-Wüst AP, Huber M, Steinhart H: Trans fatty acids in human milk are an indicator of different maternal dietary sources containing trans fatty acids. Lipids 2010;45:245-251.
Bendsen NT, Christensen R, Bartels EM, Astrup A: Consumption of industrial and ruminant trans fatty acids and risk of coronary heart disease: a systematic review and metaanalysis of cohort studies. Eur J Clin Nutr 2011;65:773-783.

41 Volpp KG, John LK, Troxel AB, Norton L, Fassbender J, Loewenstein G: Financial in centive-based approaches for weight loss: a randomized trial. JAMA 2008;300:2631-2637. 